\title{
PAPER
}

\section{Lack of hemispheric dominance for consciousness in acute ischaemic stroke}

\author{
B Cucchiara, S E Kasner, D A Wolk, P D Lyden, V A Knappertz, T Ashwood, \\ T Odergren, A Nordlund, for the CLASS-I Investigators
}

J Neurol Neurosurg Psychiatry 2003;74:889-892

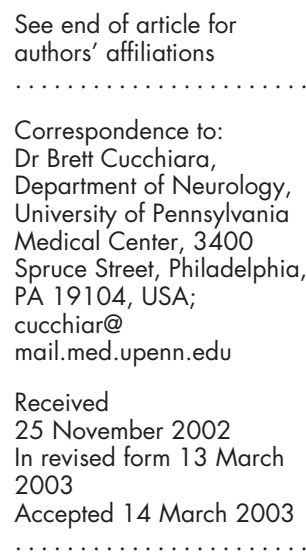

\begin{abstract}
Background: Previous reports have suggested left hemispheric dominance for maintaining consciousness, although there is controversy over this claim.

Objective: To compare early impairment of level of consciousness between patients with right and left hemispheric stroke.

Methods: Data from 564 patients with ischaemic stroke enrolled in the placebo arm of a trial of a putative neuroprotectant were analysed. All patients had major hemispheric stroke with cortical dysfunction, visual field deficit, and limb weakness, with symptom onset within 12 hours of enrolment. Patients were prospectively evaluated on a predefined scale $(1-6 ; 1=$ fully awake, higher scores representing greater impairment) to measure level of consciousness at multiple time points over the initial 24 hours after presentation. The National Institutes of Health $(\mathrm{NIH})$ stroke scale score at presentation and infarct volume at 30 days were determined.

Results: Some degree of impairment in level of consciousness was observed in 409 of the 564 patients $(73 \%)$. Median maximum sedation score was 2 for both right and left hemispheric stroke $(p=0.91)$. Mean sedation score over 24 hours was 1.5 for both right and left stroke $(p=0.75)$. There was no difference between level of consciousness scores in right and left stroke at any individual time point during the 24 hour monitoring period. No association between side and impairment in level of consciousness was seen after adjustment for stroke severity and infarct volume.

Conclusions: In contrast to previous reports, there was no evidence for hemispheric dominance for consciousness in the setting of a major hemispheric stroke.
\end{abstract}

V arious investigators have proposed that there is left hemispheric dominance for consciousness. This hypothesis is based on two major lines of evidence: greater impairment of consciousness after acute left hemispheric stroke than after acute right hemispheric stroke ${ }^{12}$; and greater sedation following left sided versus right sided intracarotid amobarbital injections given as part of the Wada test. ${ }^{3-5}$ However, other reports have called this assessment into question..$^{6-13}$ Interpretation of these conflicting data is complicated by the fact that "consciousness" is a broad term encompassing many aspects of global cognitive function, including arousal, awareness, and attention. The ideal means of measuring consciousness, and indeed the very definition of consciousness, remains controversial. Despite these obvious limitations, the concept of measuring "level of consciousness" remains firmly embedded in the landscape of clinical neurology.

To evaluate the evidence for asymmetry in consciousness following major hemispheric stroke, we analysed patients from the placebo group of a large trial of a putative neuroprotectant. Patients were enrolled within 12 hours of onset of major anterior circulation stroke, and level of consciousness was assessed prospectively at multiple time points over the first 24 hours after presentation. To measure level of consciousness we used a simple clinical scale which assessed the patients' state of arousal and ability to interact with the rater.

\section{METHODS}

Trial design

A prospective cohort of placebo treated patients from the "clomethiazole in acute stroke study-ischemic stroke" (CLASS-I) trial who fitted the Oxfordshire classification of total anterior circulation syndrome (TACS) was analysed. These patients nearly always have large hemispheric infarctions in the territory of the middle cerebral artery. A detailed description of the CLASS-I trial organisation and protocol has been published previously. ${ }^{14}$ Patients were enrolled within 12 hours of onset of major anterior circulation ischaemic stroke, defined as the presence of higher cortical dysfunction, visual field deficit, and limb weakness. Stroke severity was measured with the National Institutes of Health stroke scale (NIHSS), which quantifies key elements of the neurological examination including responsiveness, visual fields, eye movements, facial and limb strength, sensation, speech and language, ataxia, and neglect. Higher scores denote more severe deficits. ${ }^{15}$

A six point scale was used to measure the patients' level of consciousness immediately before the initiation of the study drug and then at 15,30, and 60 minutes and at 3, 6, 8, 10, 12, $15,18,21$, and 24 hours. The level of consciousness scale consisted of the following scoring system: 1, fully awake; 2, drowsy but answers when spoken to; 3 , answers slowly when spoken to; 4 , reacts when spoken to but does not answer; 5 , reacts only to pain; 6 , does not react to pain. A specific instruction was given that for dysphasic patients an obvious attempt to respond was acceptable as an answer. Normal sleep (patient fully awake after rousing) was scored 1 and entered as a comment. Patients were excluded from CLASS-I if the level of consciousness score was $\geqslant 2$ at baseline, though "slight drowsiness" was acceptable. Sedative drugs were prohibited during the study period. At one month, clinical outcome and, in survivors, infarct volume (determined by head computed tomography (CT)) were assessed.

\section{Statistical analysis}

Patients with and without impaired consciousness were compared for demographic features, pre-existing conditions, clinical characteristics, and laboratory results. The association of side with level of consciousness score was primarily assessed 
Table 1 Patient characteristics

\begin{tabular}{|c|c|c|c|c|}
\hline & All $(n=564)$ & $\begin{array}{l}\text { No impairment } \\
\text { in LOC }(n=155)\end{array}$ & $\begin{array}{l}\text { Any impairment in } \\
\text { LOC }(n=409)\end{array}$ & $\mathrm{p}$ Value \\
\hline \multicolumn{5}{|l|}{ Demographics } \\
\hline Age (years) & 71.7 (12.3) & 72.7 (11.8) & $71.4(12.4)$ & 0.26 \\
\hline Female & $52 \%$ & $50 \%$ & $53 \%$ & 0.53 \\
\hline White & $84 \%$ & $85 \%$ & $83 \%$ & 0.61 \\
\hline \multicolumn{5}{|l|}{ Risk factors } \\
\hline Previous TIA & $19 \%$ & $22 \%$ & $18 \%$ & 0.30 \\
\hline Previous stroke & $22 \%$ & $19 \%$ & $22 \%$ & 0.42 \\
\hline \multicolumn{5}{|l|}{ Clinical features } \\
\hline NIHSS score (median (IQR)) & $17(13$ to 21$)$ & 16 (12 to 20$)$ & 18 (14 to 21$)$ & 0.02 \\
\hline Left hemisphere involved & $53 \%$ & $55 \%$ & $53 \%$ & 0.74 \\
\hline Temperature on admission $\left({ }^{\circ} \mathrm{C}\right)$ & $36.8(0.7)$ & $36.7(0.8)$ & $36.8(0.7)$ & 0.40 \\
\hline Temperature after $24 \mathrm{~h}\left({ }^{\circ} \mathrm{C}\right)$ & $37.1(0.7)$ & $37.1(0.7)$ & $37.2(0.7)$ & 0.24 \\
\hline Diastolic BP (mm Hg) & $77(17)$ & $76(17)$ & $78(16)$ & 0.15 \\
\hline Systolic BP (mm Hg) & $151(24)$ & $149(23)$ & $151(25)$ & 0.35 \\
\hline \multicolumn{5}{|l|}{ Laboratory data } \\
\hline $\begin{array}{l}\text { Blood glucose on admission } \\
(\mathrm{mmol} / \mathrm{l})\end{array}$ & $7.71(3.44)$ & $7.66(3.66)$ & $7.77(3.33)$ & 0.76 \\
\hline Blood glucose at $24 \mathrm{~h}(\mathrm{mmol} / \mathrm{l})$ & $7.88(3.61)$ & $7.60(3.27)$ & 7.99 (3.72) & 0.27 \\
\hline $\begin{array}{l}\text { Infarct volume }\left(\mathrm{cm}^{3}\right) \dagger \text { (median } \\
(\text { (IQR)) }\end{array}$ & 41 (9 to 97) & 25 (4 to 64) & 48 (13 to 109) & $<0.001$ \\
\hline Mortality & $20 \%$ & $14 \%$ & $22 \%$ & 0.03 \\
\hline $\begin{array}{l}\text { Values are mean }(S D) \text { or \% unles } \\
\text { †Infarct volume available only in } \\
\mathrm{BP} \text {, blood pressure; IQR, interqu } \\
\text { Health stroke scale; } T I A \text {, transien }\end{array}$ & $\begin{array}{l}\text { ed. } \\
\text { vors at } 30 \text { days } \\
\text { range; } L O C \text {, le } \\
\text { emic attack. }\end{array}$ & $\begin{array}{l}n=421 \\
l \text { of consciousne }\end{array}$ & NIHSS, National I & itutes of \\
\hline
\end{tabular}

using maximum level of consciousness score over the 24 hour monitoring period. A secondary analysis using scores at individual time points and a mean level of consciousness score over the 24 hour monitoring period was also done.

In the bivariate analyses, dichotomous or categorical variables were compared with the $\chi^{2}$ test, and continuous variables were compared with the $t$ test or Wilcoxon rank sum test, as appropriate. All tests were two sided. Variables were considered for multivariate analysis if they were associated with level of consciousness in the bivariate analysis at a probability level of $<0.10$. Multivariate analyses were done using ordered logistic regression with maximum level of consciousness score as the dependent variable, and were adjusted for age, sex, and race, as these were considered to be potentially confounding variables a priori. This analysis was repeated substituting mean level of consciousness score over 24 hours and level of consciousness score at each individual time point as the dependent variables. In the final model, an association was considered significant at a probability $(p)$ value of $<0.05$.

All analyses were done using STATA version 6.0 (STATA Corporation, College Station, Texas, USA).

\section{RESULTS}

\section{Patient characteristics}

In all, 599 patients were randomised to the placebo arm of CLASS-I; in 15 of these patients, treatment was never started and the collection of level of consciousness scores was therefore incomplete. Of the 584 remaining patients, 564 (96\%) had a major anterior circulation stroke consistent with the Oxfordshire TACS classification. Their mean (SD) age was 72 (12) years, and 52\% were female. Medical history included habitual smoking in 19\%, diabetes mellitus in $25 \%$, hypertension in $76 \%$, previous stroke in $22 \%$, and previous transient ischaemic attacks (TIA) in 19\%. Mean and median NIHSS scores were 17. Mean time from symptom onset to placebo administration was 7.7 (2.7) hours. At one month, 114 patients (20\%) had died. The majority of patients (73\%) experienced some degree of impairment in level of consciousness over the monitoring period (level of consciousness score $>1$ on at least one examination); patients with such impairment had higher baseline NIHSS scores $(p=0.02)$, greater 30 day mortality $(p=0.03)$, and, in survivors, a larger infarct volume $(\mathrm{p}<0.001)$. These data are summarised in table 1 .

\section{Side differences}

At the time of presentation, stroke involved the right hemisphere in 261 patients (46\%) and the left in 299 (53\%). In four patients $(0.7 \%)$ stroke laterality was unknown, missing, or bilateral, and these patients were excluded from further analysis. Median NIHSS score was 15 for right and 19 for left hemispheric stroke $(\mathrm{p}<0.0001)$. Median total motor score (NIHSS items $5+6$ ) was 6 (mean $5.7(2.3)$ ) with right hemisphere stroke, and 7 (mean $6.0(2.4)$ ) with left hemisphere stroke $(p=0.19)$. Neglect (NIHSS item $11 \geqslant 1)$ was present to some degree in $95 \%$ of right and $70 \%$ of left hemisphere strokes $(p<0.001)$. Aphasia (NIHSS item $9 \geqslant 1$ ) was present to some degree in $96 \%$ of left and $20 \%$ of right hemisphere strokes $(\mathrm{p}<0.001)$.

Infarct volume at 30 days was available in 421 of the 451 survivors $(93 \%)$, and tended to be greater on the right (median $47 \mathrm{~cm}^{3}$ ) than on the left (median $36 \mathrm{~cm}^{3}$ ), though this was not statistically significant $(p=0.12)$. These differences are summarised in table 2. There were no significant differences between patients with right and left hemisphere strokes in history of diabetes, hypertension, smoking, or previous stroke or TIA.

Given the surprising number of right hemispheric strokes with aphasia and left hemispheric strokes with neglect, further analysis of these subgroups was undertaken. Infarct volume in right hemispheric stroke patients with aphasia was greater than in those without aphasia (mean (SD), 94 (87) $v$ $\left.66(67) \mathrm{cm}^{3}\right)$, though this was not statistically significant $(\mathrm{p}=0.14)$. Of the 53 aphasic right hemisphere stroke patients, NIHSS item 9 score was 1 (mild to moderate aphasia) in $70 \%, 2$ (severe aphasia) in 19\%, and 3 (mute or global aphasia) in $11 \%$; in aphasic left hemispheric stroke patients, these percentages were $10 \%, 41 \%$, and $49 \%$, respectively. The finding that patients with aphasic right hemispheric stroke 
Table 2 Differences between left and right hemispheric stroke

\begin{tabular}{llll}
\hline & Left hemisphere $(\mathrm{n}=299)^{*}$ & Right hemisphere $(\mathrm{n}=261)^{*}$ & $\mathrm{p}$ Value \\
\hline Total NIHSS (median (IQR)) & $19(16$ to 23$)$ & $15(12$ to 18$)$ & $<0.0001$ \\
Aphasia† & $96 \%$ & $20 \%$ & $<0.001$ \\
Neglectł & $70 \%$ & $95 \%$ & $<0.001$ \\
Total motor score (median (IQR)) & $7(4$ to 8$)$ & $47(11$ to 114$)$ & 0.19 \\
Infarct volume $\left(\mathrm{cm}^{3}\right)$ I (median (IQR)) & $36(8$ to 82$)$ & $2(1$ to 2$)$ & 0.12 \\
LOC score maximum (median (IQR)) & $2(1$ to 2$)$ & $1.5(0.4)$ & 0.91 \\
LOC score (mean (SD)) & $1.5(0.4)$ & $18.8 \%(\mathrm{n}=49)$ & 0.75 \\
Mortality & $21.4 \%(\mathrm{n}=64)$ & & 0.44 \\
\hline
\end{tabular}

* Patients in whom stroke was bilateral or side unknown were excluded.

†Score $\geqslant 1$ NIHSS, item 9.

$\ddagger$ Score $\geqslant 1$ NIHSS, item 11 .

IAs measured by head computed tomography at 30 days, only available for survivors $(n=421)$.

IQR, interquartile range; LOC, level of consciousness; NIHSS, National Institutes of Health stroke scale.

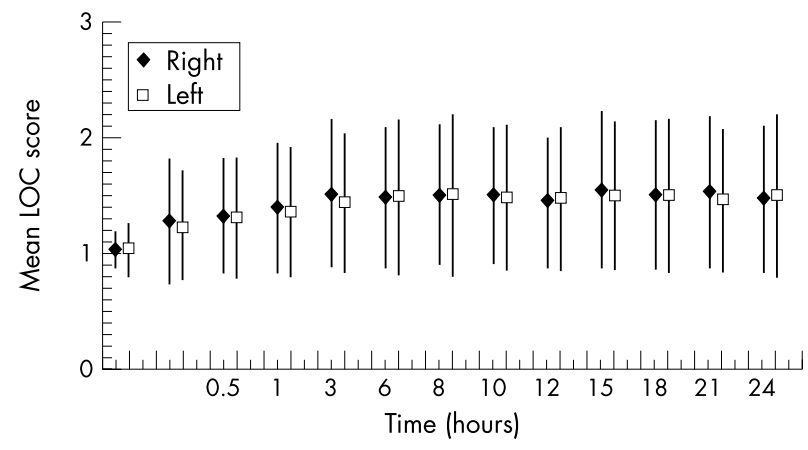

Figure 1 Mean level of consciousness (LOC) score at individual time points for right and left hemispheric stroke.

had milder aphasia than those with left hemispheric stroke was statistically significant $(p<0.001)$. Left hemispheric stroke patients with neglect were more likely to have a gaze preference or forced gaze deviation (NIHSS item $2 \geqslant 1$ ) than those without neglect $(72 \% v 56 \%, \mathrm{p}=0.01)$. There was no difference in infarct volume between left hemispheric stroke patients with and without neglect. In patients with neglect, there was no significant difference between left and right hemispheric stroke in neglect severity.

\section{Level of consciousness and affected side}

Median maximum level of consciousness score during the first 24 hours was 2 for right and 2 for left hemispheric stroke $(\mathrm{p}=0.91)$. Mean level of consciousness score was 1.5 for both right and left hemisphere stroke $(\mathrm{p}=0.73)$. Analysis at each individual time point over the 24 hour monitoring period showed no significant difference at any time between right and left hemispheric stroke (fig l).

After adjustment for age, sex, race, and stroke severity (total NIHSS score), there was a modest non-significant trend toward greater maximum level of consciousness score with right hemisphere stroke (odds ratio 1.20; 95\% confidence interval, 0.96 to $1.49 ; \mathrm{p}=0.11)$. Given the established hemispheric bias in the NIHSS caused by greater weighting of language disturbances than neglect, ${ }^{16}$ we undertook additional exploratory analyses substituting NIHSS total motor score-defined as the sum of the scores from NIHSS items 5 and 6-for total NIHSS score. There was no association between side and maximum level of consciousness score in this analysis $(p=0.92)$. Analysis of those patients for whom infarct volume was available showed no association between maximum level of consciousness score and side after adjustment for age, sex, race, and infarct volume $(\mathrm{p}=0.34)$. Analysis of patients for whom infarct volume was not determined also showed no association between side and level of consciousness $(\mathrm{p}=0.54)$.
Similar findings were observed in all the above analyses when mean level of consciousness score over 24 hours and level of consciousness score at each individual time point were substituted as the dependent variables in place of maximum consciousness score.

\section{DISCUSSION}

There is much controversy over whether there is hemispheric dominance for maintaining consciousness. A retrospective clinicopathological study of 100 patients with hemispheric stroke found that early alterations of consciousness were more severe with lesions involving the dominant hemisphere. ${ }^{1} \mathrm{~A}$ smaller study of 47 patients found initial impairment of consciousness in $57 \%$ of patients with left hemispheric stroke compared with $25 \%$ of patients with right hemispheric stroke. ${ }^{2}$ These studies were done before the introduction of modern neuroimaging, lacked standardised measures of stroke severity and level of consciousness, and did not control for time from symptom onset to examination. Moreover, the latter study used several verbal determinants of arousal.

Intracarotid amobarbital injections in the left carotid have been shown to result in greater impairment of arousal than right sided injections, based on clinical observation, formal measurement tools such as the Glasgow coma scale, and tests of continuous visual and auditory vigilance. ${ }^{3-5}$ Further, left sided injections cause more prolonged EEG slowing and a greater decrease in cerebral blood flow in the injected hemisphere than right sided injections. ${ }^{17}{ }^{18}$ Whether hemispheric asymmetry in the intracarotid amobarbital test indicates true hemispheric dominance for arousal is controversial; proposed alternative explanations for a differential effect include increased cross flow to the opposite hemisphere following left versus right amobarbital injection ${ }^{9}{ }^{19}$ or differential hemispheric metabolic demands. ${ }^{20}$

In contrast, there is also experimental evidence in support of right hemispheric dominance for consciousness. Following right carotid amobarbital injection, patients' subjective experience of alertness ${ }^{12}{ }^{13}$ and measures of focused attention, such as tests of visual scanning and directed eye gaze, ${ }^{9-11}$ are more impaired than after left sided injection. When compared with left brain injured patients, right brain injured patients have hypoarousal, as measured by galvanic skin responses to local application of stimuli ipsilateral to the damaged hemisphere, reduced cerebral activation with prolonged reaction times during both single and multiple simultaneous tasks, and greater electroencephalographic slowing. ${ }^{6-8}$ Based on these and similar experimental findings, researchers studying neglect have proposed a right hemisphere dominant attention-arousal-intention system through cortico-limbicreticular connections, and suggested that the right hemisphere plays a greater role in regulating the reticular activating system than the left. ${ }^{21}$ 
In this study of patients with acute major hemispheric stroke, we found that impaired level of consciousness was frequent. However, we did not find evidence of hemispheric asymmetry in early impairment of level of consciousness. Strengths of our study include the large number of patients evaluated; the matching of stroke location between right and left sided stroke by inclusion of only patients with anterior circulation stroke meeting the Oxfordshire TACS classification; the prospective nature of data collection; the use of a standardised measurement tool to assess level of consciousness and stroke severity; and assessments of level of consciousness at multiple time points. In addition, patients were studied within a standardised period of time from symptom onset $(<12$ hours).

A surprisingly large number of right hemispheric stroke patients in our study were reported to have aphasia (20\%), and a surprisingly large number of left hemispheric stroke patients to have neglect $(70 \%)$, as determined by the NIHSS score. The explanation for this finding is unclear. The NIHSS assessment of aphasia is relatively simplistic and rapid, and is not equivalent to a comprehensive neurocognitive examination. A portion of the NIHSS aphasia examination consists of describing the events taking place in a relatively complex picture. It is possible that specific types of cognitive impairment often seen in right hemispheric stroke, such as deficits in visuospatial processing, confusional states, and disorders of prosody, may have created a mild impairment in verbal communication and thus been scored as aphasia on the NIHSS. Consistent with this possibility is the finding that aphasic patients with right hemispheric stroke generally were scored as having only a mild aphasia, in contrast to left hemispheric stroke patients.

The high prevalence of reported neglect in our left hemispheric stroke patients is probably explained by the fact that these patients had major acute hemispheric stroke with associated visual field deficits and frequent eye deviation. Despite the NIHSS having an explicit caution on the evaluation of neglect in patients with a visual field defect, the need for rapid scoring in the setting of an acute treatment trial and the limitations on examination imposed by the presence of aphasia probably resulted in an overestimation of neglect. This highlights a potentially significant inaccuracy in the use of the NIHSS in patients with major hemispheric strokes.

A notable limitation of our study was the exclusion of patients with significantly impaired consciousness at presentation. This might have obscured a true hemispheric asymmetry by selectively excluding those patients with greater degrees of very early impairment in their level of consciousness. We think this is unlikely for several reasons. First, our patients were equally divided between right and left hemispheric stroke, arguing against a systematic bias favouring enrolment of a particular affected hemisphere. Second, assessment of consciousness at a single time point (that is, enrolment only) is problematic given that numerous factors besides the structural brain injury are likely to influence a patient's level of arousal, including time of day, concurrent medical illness, and the medical staff's perceived need to stimulate the patient to assess their condition. Decreased level of consciousness in the setting of hemispheric stroke often develops owing to progressive oedema and infarct extension in the initial days following stroke onset. Measurement of level of consciousness must therefore take into account the time from symptom onset to assessment. Level of consciousness in our patients was assessed very early after symptom onset and on multiple occasions, minimising the impact of such factors. Finally, and most importantly, the patients with left and right sided stroke were well matched in terms of stroke severity as measured by the NIHSS total motor score, presence of cortical signs, and, most convincingly, CT measurements of infarct volume in survivors.

It is important to recognise that "consciousness" means different things to different people. Previous investigators have suggested that left/right cerebral differences in consciousness may be more qualitative than quantitative, reflecting the specialised features of lateralised hemispheric function, and that it is imprecise to equate arousal, attention, and consciousness. ${ }^{22}$ Our study suggests that altered consciousness is common after hemispheric stroke but that hemispheric asymmetry is not apparent when a general measure of level of consciousness (as represented by our scale) is used. Further studies exploring exactly what facets of "consciousness" are affected with lateralised hemispheric injury may expand our understanding of hemispheric specialisation and the neural networks underlying awareness.

\section{Authors' affiliations}

B Cucchiara, S E Kasner, Department of Neurology, University of Pennsylvania Medical Center, Philadelphia, USA

D A Wolk, Brigham and Women's Hospital, Division of Cognitive and Behavioral Neurology, Boston, Massachusetts, USA

P D Lyden, Department of Neurology, University of California-San Diego, San Diego, California, USA

V Knappertz, Specialized Therapeutics, Montville, New Jersey, USA

T Ashwood, AstraZeneca Pharmaceuticals, Södertälie, Sweden

A Nordlund, Centre for Work and Rehabilitation, Faculty of Health,

Linköping University, Linköping, Sweden

T Odergren, AstraZeneca Pharmaceuticals, Wilmington, Delaware, USA

\section{REFERENCES}

1 Schwartz B. Hemispheric dominance and consciousness. Acta Neurol Scand 1967:43:513-25.

2 Albert ML, Silverberg R, Reches A, et al. Cerebral dominance for consciousness. Arch Neurol 1976;33:453-4.

3 Meador KJ, Loring DW, Lee GP, et al. Level of consciousness and memory during the intracarotid sodium amobarbital procedure. Brain Cogn 1997;33:178-88.

4 Serafetinides EA, Hoare RD, Driver MV. Intracarotid sodium amylobarbitone and cerebral dominance for speech and consciousness. Brain 1965;88:107-30

5 Glosser G, Cole LC, Deutsch GK, et al. Hemispheric asymmetries in arousal affect outcome of the intracarotid amobarbital test. Neurology 1999;52:1583-90.

6 Heilman KM, Schwartz HD, Watson RT. Hypoarousal in patients with the neglect syndrome and emotional indifference. Neurology 1978;28:229-32

7 Coslett HB, Bowers D, Heilman KM. Reduction in cerebral activation after right hemisphere stroke. Neurology 1987;37:957-62.

8 Watson RT, Andriola M, Heilman KM. The electroencephalogram in neglect. J Neurol Sci 1977;34:343-8.

9 Huh K, Meador KJ, Loring DW, et al. Attentional mechanisms during the intracarotid amobarbital test. Neurology 1989;39:1183-6.

10 Meador KJ, Loring DW, Lee GP, et al. Hemisphere asymmetry for eye gaze mechanisms. Brain 1989;112:103-11.

11 Spiers $\mathbf{P}$, Schomer D, Blume $\mathrm{H}$, et al. Visual neglect during intracarotid amobarbital testing. Neurology 1990:40:1600-6.

12 Gilmore R, Heilman K, Schmidt R, et al. Anosognosia during Wada testing. Neurology 1992;42:925-7.

13 Durkin MW, Meador KJ, Nichols ME, et al. Anosognosia and the intracarotid amobarbital procedure (Wada test). Neurology 1994:44:978-9

14 Lyden P, Shuaib A, Ng K, et al. Clomethiazole acute stroke study in ischemic stroke (CLASS-I): final results [editorial comment]. Stroke 2002;33: 122-9.

15 Lyden P, Brott T, Tilley B, et al. Improved reliability of the NIH stroke scale using video training. Stroke 1994;25:2220-6.

16 Fink JN, Selim MH, Kumar S, et al. Is the Association of National Institutes of Health stroke scale scores and acute magnetic resonance imaging stroke volume equal for patients with right- and left-hemisphere ischemic stroke? Stroke 2002;33:954-8.

17 McMackin D, Jones-Gotman M, Dubeau F, et al. Regional cerebral blood flow and language dominance: SPECT during intracarotid amobarbital testing. Neurology 1998;50:943-50.

18 Biersack HJ, Linke D, Brassel F, et al. Technetium-99m HM-PAO brain SPECT in epileptic patients before and during unilateral hemispheric anesthesia (Wada test): report of three cases. J Nucl Med 1987;28: 1763-7

19 Perrine K, Devinsky O, Luciano DJ, et al. Correlates of arterial-filling patterns in the intracarotid amobarbital procedure. Arch Neurol 1995:52:712-16.

20 Frumkin LR, Grim P. Is there pharmacological asymmetry in the human brain? An hypothesis for the differential hemispheric action of barbiturates. Int J Neurosci 1981;13:187-97.

21 Heilman KM, Valenstein E, Watson RT. Neglect and related disorders. Semin Neurol 2000;20:463-70.

22 Meador KJ, Loring DW. The Wada test: controversies, concerns, and insights. Neurology 1999;52:1535-6. 